\title{
Theory of the zero-bias anomaly in magnetic tunnel junctions: Inelastic tunneling via impurities
}

\author{
L. Sheng, ${ }^{1}$ D. Y. Xing, ${ }^{1}$ and D. N. Sheng ${ }^{2}$ \\ ${ }^{1}$ National Laboratory of Solid State Microstructures, Nanjing University, Nanjing 210093, China \\ ${ }^{2}$ Department of Physics and Astronomy, California State University, Northridge, California 91330, USA
}

(Received 12 April 2004; published 22 September 2004)

\begin{abstract}
Using the closed-time path-integral approach, we nonperturbatively study inelastic tunneling of electrons via magnetic impurities in the barrier accompanied by phonon emission in a magnetic tunnel junction. The spectrum density of phonon emission is found to show a power-law infrared singularity $\sim \omega^{-(1-g)}$ with $g$ the dimensionless electron-phonon coupling. As a consequence, the tunneling conductance $G(V)$ increases with bias voltage $|V|$ as $G(V)-G(0) \sim|V|^{2 g}$, exhibiting a discontinuity in slope at $V=0$ for $g \leqslant 0.5$. This theory can reproduce both cusplike and noncusplike features of the zero-bias anomaly of tunneling resistance and magnetoresistance widely observed in experiments.
\end{abstract}

DOI: 10.1103/PhysRevB.70.094416

PACS number(s): 75.47.-m, 72.25.Ba, 72.10.Di, 73.40.Rw

The tunneling magnetoresistance (TMR) effect in a magnetic junction composed of two ferromagnetic (FM) electrodes separated by an insulating layer is presently of great interest because of its potential for device applications such as random access memories and magnetic sensors. ${ }^{1-11}$ While the TMR effect has been attributed to spin polarization of conduction electrons in the FM electrodes, ${ }^{1-3}$ its novel voltage dependence is still puzzling. The tunneling resistance and TMR ratio generally decrease with increasing bias voltage, often exhibiting a cusplike feature at zero bias. ${ }^{1,5-11}$ This effect, usually called the zero-bias anomaly (ZBA), ${ }^{6}$ was not only widely observed in transition-metal magnetic junctions, but also exists in semiconductor GaMnAs/AlAs/GaMnAs magnetic junctions. $^{12}$

It is found that the ZBA is very sensitive to the insulating material of the barrier. In earlier measurements ${ }^{1}$ on $\mathrm{Fe} / \mathrm{Ge} / \mathrm{Co}$ junctions, the characteristic voltage $V_{1 / 2}$ that halves the TMR ratio is about $3 \mathrm{mV}$. It was reported recently that for $\mathrm{FM} / \mathrm{Al}_{2} \mathrm{O}_{3} / \mathrm{FM}$ with $\mathrm{Fe}, \mathrm{Co}, \mathrm{Ni}$, or their alloys as the FM electrodes, $V_{1 / 2}$ is usually one hundred to several hundred $\mathrm{mV},{ }^{5-9}$ and for $\mathrm{Ni} / \mathrm{NiO} / \mathrm{Co}$ junctions $V_{1 / 2}$ is a few ten $\mathrm{mV} .{ }^{11}$ Junctions made of the same FM electrodes and identical but differently prepared insulator vary considerably concerning the voltage dependence of the resistance and TMR. ${ }^{9}$ It was reported that the ZBA in the semiconductor magnetic tunnel junctions is much more striking with $V_{1 / 2}$ about $3 \mathrm{mV} .{ }^{12}$ Interestingly, in a very recent experiment ${ }^{13}$ of the TMR between a Co sample and a CoFeSiB tip through a vacuum barrier, the ZBA did not occur for bias voltage up to a volt.

Understanding of the ZBA is not only important for optimizing the TMR in practical applications, but also provides critical insight into the underlying physics of spin-dependent transport. There exist several possible explanations for the anomaly. The simplest one may involve the energy dependence of the electron density of states (DOS) and elastic transmission matrix elements. ${ }^{3,14,15}$ Unfortunately, by comparison with experimental data, the TMR ratio calculated from such a model decreases too slowly at low bias. ${ }^{7}$ The second mechanism is that the hot electrons from the emitting electrode may be scattered by local magnetic moments at the interfaces through $s-d$ interaction, ${ }^{6}$ which contributes to the conductance a term $\Delta G \sim|V|$ at low bias voltage $V$. This theory succeeded in explaining some experimental data. The third mechanism might be based upon the electron tunneling assisted by magnons and phonons, ${ }^{7,16-18}$ which was found to contribute $\Delta G \sim|V|^{3 / 2}$ for FM magnons, $|V|^{2}$ for surface antiferromagnetic magnons, and $|V|^{4}$ for phonons. This mechanism may explain some experimental data without the cusplike feature at zero bias, ${ }^{18}$ but has the difficulty to interpret the cusplike feature of the ZBA observed in many other experiments. ${ }^{5-7,10,12}$ Contrary to these intrinsic mechanisms, the effect of extrinsic nonmagnetic and magnetic impurities was emphasized in many works. ${ }^{8,10,11,14,18-23}$ The voltage dependence caused by elastic electron tunneling via impurities was studied theoretically in a few works. ${ }^{11,14,21}$ However, none of them reproduced the essential features of the ZBA. Based upon the results of an earlier theory, ${ }^{24}$ some authors ${ }^{10,23}$ argued that, in the presence of inelastic scattering, while single-impurity tunneling processes are still elastic, electron tunneling via multiple-impurity chains may contribute an inelastic power-law conductance $\Delta G \sim|V|^{p}$ with $p=\frac{4}{3}\left(\frac{5}{2}\right)$ for two-impurity (three-impurity) processes. Presently, the relevant mechanism for the ZBA is still under debate.

In this paper, a combined effect of the magnetic impurities in the barrier and the electron-phonon interaction is proposed as a possible mechanism for the ZBA. What we consider in this model is not a simple addition of the elastic tunneling of electrons via the impurities and the inelastic one assisted by phonons, ${ }^{18}$ but a novel inelastic electron tunneling due to the strong interplay between them, in which the electron tunneling via impurities is always accompanied by phonon emission. It is found that the spectrum density of phonon emission has a power-law infrared singularity $\sim \omega^{-(1-g)}$ with $g$ the dimensionless electron-phonon coupling. As a consequence, the conductance via impurities 
$G^{\mathrm{I}}(V)$ obeys a power-law voltage dependence $G^{\mathrm{I}}(V) \sim|V|^{2 g}$ with a discontinuity in slope at $V=0$ for $g \leqslant 0.5$. The conductance exponent $2 g$ may be either smaller or greater than unity, being different from the earlier theories, $6,16-18,24$ where the conductance exponents are never smaller than unity. The calculated results can reproduce both cusplike and noncusplike features of ZBA observed in experiments. An experiment based upon the isotope effect is suggested to further verify this theory.

The model Hamiltonian of a magnetic tunnel junction is written as $H=H_{\mathrm{L}}+H_{\mathrm{R}}+H_{\mathrm{C}}+H_{\mathrm{T}}$. Here, $H_{\mathrm{L}(\mathrm{R})}$ $=\Sigma_{k s} \epsilon_{k s}^{\mathrm{L}(\mathrm{R})} a_{k s}^{\mathrm{L}(\mathrm{R}) \dagger} a_{k s}^{\mathrm{L}(\mathrm{R})}$ is the Hamiltonian of the electrons in the left (right) electrodes. The third term is the Hamiltonian of the electrons on the magnetic impurities,

$$
\begin{aligned}
H_{\mathrm{C}}= & \sum_{l s s^{\prime}}\left(E_{0} \delta_{s s^{\prime}}-J \boldsymbol{\sigma}_{s s^{\prime}} \cdot \mathbf{S}_{l}\right) d_{l s}^{\dagger} d_{l s^{\prime}}+\sum_{q \lambda} \omega_{q \lambda} b_{q \lambda}^{\dagger} b_{q \lambda} \\
& +\sum_{q \lambda l s} M_{q \lambda} e^{i \mathbf{q} \cdot \mathbf{R}_{l}}\left(b_{q \lambda}+b_{-q \lambda}^{\dagger}\right) d_{l s}^{\dagger} d_{l s} .
\end{aligned}
$$

Here, $d_{l s}$ annihilates an electron with spin $s$ on magnetic impurity $l$, and $b_{q \lambda}$ annihilates an acoustic phonon of wave vector $q$ and polarization $\lambda$ in the system. The magnetic impurities in the barrier are presumably the atoms of FM metals of the electrodes mixed into the barrier. Some of their outer-shell electrons are itinerant, and the others form localized spins. $J$ represents the $s$ - $d$-like interaction between the itinerant electrons and localized spin $\mathbf{S}_{l}$ with $\hat{\boldsymbol{\sigma}}$ the Pauli matrix. The spins of the transition-metal ferromagnets under consideration are usually greater than $\frac{1}{2}$, and can be treated as classical approximately. Since the impurities are also a part of the lattice, electrons scattering with the impurities will generally cause emission or absorption of phonons of the system, which is described by the electron-phonon interaction of the last term in Eq. (1). It is worth mentioning that a similar impurity-mediated electronphonon interaction has been used to account for the leading
$T^{2}$ correction to residue resistivity of simple metals. ${ }^{25,26}$ Here, the direct scattering of electrons by phonons is neglected, which was found to open new tunneling channels, and yield even, positive correction to the conductance. ${ }^{27}$ It does not account for the ZBA. The electron tunneling Hamiltonian is given by

$$
\begin{aligned}
H_{\mathrm{T}}= & \sum_{k k^{\prime} s}\left(T_{k k^{\prime} s}^{\mathrm{D}} a_{k s}^{\mathrm{L} \dagger} a_{k^{\prime} s}^{\mathrm{R}}+\text { H.c. }\right)+\sum_{l k s}\left(T_{l k s}^{\mathrm{IL}} a_{k s}^{\mathrm{L} \dagger} d_{l s}+\text { H.c. }\right) \\
& +\sum_{l k s}\left(T_{l k s}^{\mathrm{IR}} a_{k s}^{\mathrm{R} \dagger} d_{l s}+\text { H.c. }\right)
\end{aligned}
$$

where the first term represents direct tunneling of electrons between the electrodes, and the second and third terms represent transfer of electrons between the electrodes and the impurities.

To treat the electron-phonon interaction in a nonperturbative manner, the Lang-Firsov transformation $e^{\mathcal{S}}$ with $\mathcal{S}=$

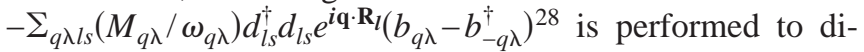
agonalize the electron-phonon interaction. The transformed Hamiltonian $e^{\mathcal{S}} H e^{-\mathcal{S}}$ is the same as the original one, except that the last term in $H_{\mathrm{C}}$ vanishes, and operators $d_{l s}$ and $d_{l s}^{\dagger}$ in $H_{\mathrm{T}}$ are replaced with $d_{l s} X_{l}$ and $d_{l s}^{\dagger} X_{l}^{\dagger}$, respectively, with $X_{l}$

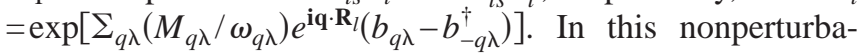
tive treatment, we see clearly that electron tunneling via the impurities causes phonon emission or absorption, which reversely renormalizes the electron tunneling matrix elements via the impurities.

The path-integral formalism on the closed-time path ${ }^{29,30}$ is employed to study this nonequilibrium problem. By integrating out the electron variables in the two electrodes, the retarded (advanced) Green's function for electrons on impurity $l$ is obtained in the frequency domain as $\hat{G}_{l, r(a)}^{\mathrm{C}}(\omega)=\left[\omega-E_{0}\right.$ $\left.+J \hat{\boldsymbol{\sigma}} \cdot \mathbf{S}_{l}-\hat{\Sigma}_{l, r(a)}(\omega)\right]^{-1}$. Here, a $2 \times 2$ matrix representation in electron spin space has been employed. The retarded selfenergy $\hat{\Sigma}_{l, r}(\omega)=\hat{\Sigma}_{l, r}^{\mathrm{L}}(\omega)+\hat{\Sigma}_{l, r}^{\mathrm{R}}(\omega)$ is a diagonal matrix with diagonal elements given by

$$
\sum_{l s, r}^{\mathrm{L}(\mathrm{R})}(\omega)=\frac{1}{2} \rho_{s}^{\mathrm{L}(\mathrm{R})}\left|T_{l}^{\mathrm{IL}(\mathrm{R})}\right|^{2} \int d \omega^{\prime}\left\{\left[1-f_{\mathrm{L}(\mathrm{R})}\left(\omega+\omega^{\prime}\right)\right] B_{>}\left(-\omega^{\prime}\right)+f_{\mathrm{L}(\mathrm{R})}\left(\omega+\omega^{\prime}\right) B_{>}\left(\omega^{\prime}\right)\right\}
$$

and the advanced self-energy $\hat{\Sigma}_{l, a}(\omega)=\left[\hat{\Sigma}_{l, r}(\omega)\right]^{\dagger}$. Here, as usual, the momentum and spin dependence in the transmission matrix element $T_{l k s}^{\mathrm{IL}(\mathrm{R})}$ is neglected. ${ }^{6} \rho_{s}^{\mathrm{L}(\mathrm{R})}$ and $f_{\mathrm{L}(\mathrm{R})}(\omega)$ are the electron DOS and Fermi distribution function of the left (right) electrode, respectively. $B_{>}(\omega)$ is the Fourier transform of $-i\left\langle X_{l}(t) X_{l}^{\dagger}(0)\right\rangle$, whose general expression can be found in Ref. 26.

The tunneling current $I$ is calculated from the rate of the charges flowing out of the emitting electrode. The total conductance $G(V)=I / V$ is obtained as $G(V)=G^{\mathrm{D}}+G^{\mathrm{I}}(V)$, where $G^{\mathrm{D}}=2 \pi e^{2}\left|T^{\mathrm{D}}\right|^{2} \operatorname{Tr}\left(\hat{\rho}^{\mathrm{L}} \hat{\rho}^{\mathrm{R}}\right)$ is the direct tunneling conductance independent of bias voltage, and

$$
\begin{aligned}
G^{\mathrm{I}}(V)= & -\frac{N_{\mathrm{I}} e}{2 \pi V} \int d \epsilon \int d \omega_{1} \int d \omega_{2} B_{>}\left(\omega_{1}\right) B_{>}\left(\omega_{2}\right)\left\{f_{\mathrm{L}}\left(\epsilon+\omega_{1}\right)\left[1-f_{\mathrm{R}}\left(\epsilon-\omega_{2}\right)\right]\right. \\
& \left.-f_{\mathrm{R}}\left(\epsilon+\omega_{1}\right)\left[1-f_{\mathrm{L}}\left(\epsilon-\omega_{2}\right)\right]\right\} \overline{\left|T_{l}^{\mathrm{IL}} T_{l}^{\mathrm{IR}}\right|^{2} \operatorname{Tr}\left[\hat{\rho}^{\mathrm{L}} \hat{G}_{l, r}^{\mathrm{C}}(\epsilon) \hat{\rho}^{\mathrm{R}} \hat{G}_{l, a}^{\mathrm{C}}(\epsilon)\right]},
\end{aligned}
$$




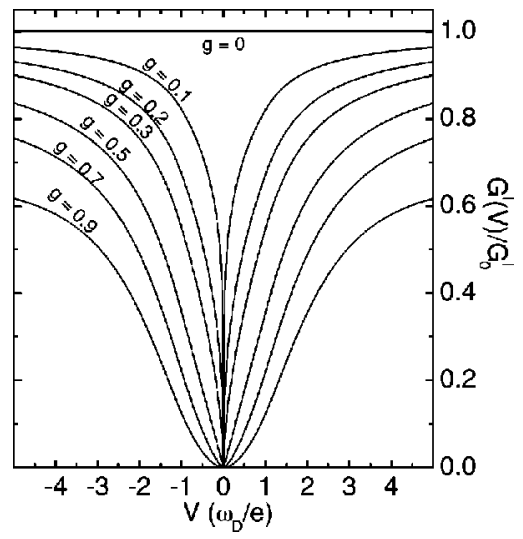

FIG. 1. Normalized impurity conductance of nonmagnetic tunnel junctions as a function of bias voltage at zero temperature. Here $J=0$ and $\rho_{\uparrow}=\rho_{\downarrow}$.

comes from the tunneling via $N_{\mathrm{I}}$ impurities. The bias dependence of the tunneling conductance is embodied in $G^{\mathrm{I}}(V)$. In Eq. (4), the last factor is averaged over the positions and spin orientations of the impurities, $\hat{\rho}^{\mathrm{L}}=\left[\left(\rho_{\uparrow}+\rho_{\downarrow}\right)+\left(\rho_{\uparrow}-\rho_{\downarrow}\right) \hat{\sigma}_{z}\right] / 2$, and $\hat{\rho}^{\mathrm{R}}=\left[\left(\rho_{\uparrow}+\rho_{\downarrow}\right) \pm\left(\rho_{\uparrow}-\rho_{\downarrow}\right) \hat{\sigma}_{z}\right] / 2$ with sign + for parallel alignment and - for antiparallel alignment. The two electrodes are considered of the same material with electron $\operatorname{DOS} \rho_{\uparrow}$ and $\rho_{\downarrow}$ for majority and minority spins, respectively.

There are two distinct resonant energies $E_{-}=E_{0}-J S$ and $E_{+}=E_{0}+J S$, at which the tunneling conductance given in Eq. (4) will be substantially enhanced. In this work, we confine ourselves to the off-resonance case, which is more probable in reality. The energy differences $\left|E_{\mathrm{F}}-E_{ \pm}\right|$with $E_{\mathrm{F}}$ the Fermi level are considered to be much greater than the broadenings of the resonant states. Besides, the bias voltage $V$ is also relatively small, $|e V|<\left|E_{\mathrm{F}}-E_{ \pm}\right|$. In this case, $\hat{\Sigma}_{l, r(a)}(\epsilon)$ in $\hat{G}_{l, r(a)}^{\mathrm{C}}(\epsilon)$ can be neglected. The last factor in Eq. (4) is then divided into two parts: $\overline{\left|T_{l}^{\mathrm{IL}} T_{l}^{\mathrm{IR}}\right|^{2}}$ and $\operatorname{Tr}\left[\hat{\rho}^{\mathrm{L}} \hat{G}_{l, r}^{\mathrm{C}}(\epsilon) \hat{\rho}^{\mathrm{R}} \hat{G}_{l, a}^{\mathrm{C}}(\epsilon)\right]$. The former is a constant after being averaged over the impurity position $\mathbf{R}_{l}$, and the latter involves only the average over the orientation of $\mathbf{S}_{l}$. The energy of the magnetic field used to produce the parallel alignment of the FM electrodes, usually several ten or hundred oersteds $\left(g_{\mathrm{L}} \mu_{\mathrm{B}} S B \lesssim 0.01 \mathrm{meV}\right)$, is much smaller than the entropy gain due to randomizing an impurity spin even at experimentally low temperatures, e.g., $1 \mathrm{~K}\left[k_{B} T \ln (2 S+1) \sim 0.1 \mathrm{meV}\right]$. Therefore, it is assumed that the impurity spins are randomly oriented for both parallel and antiparallel alignments. Moreover, one can verify that the results will not change significantly, if the magnetic field is strong enough to align all the spins ferromagnetically in the parallel alignment.

The function $B_{>}(\omega)$ represents the spectrum density of phonon emission and absorption. For simplicity, the three-dimensional Debye model is used to describe the acoustic phonons. A dimensionless electron-phonon coupling constant $g$ is defined as $g=3 \Sigma_{q \lambda}\left|M_{q \lambda}\right|^{2} \omega_{q \lambda} / \omega_{\mathrm{D}}^{3}$ with $\omega_{\mathrm{D}}$ the Debye frequency. Thus, at zero temperature we have

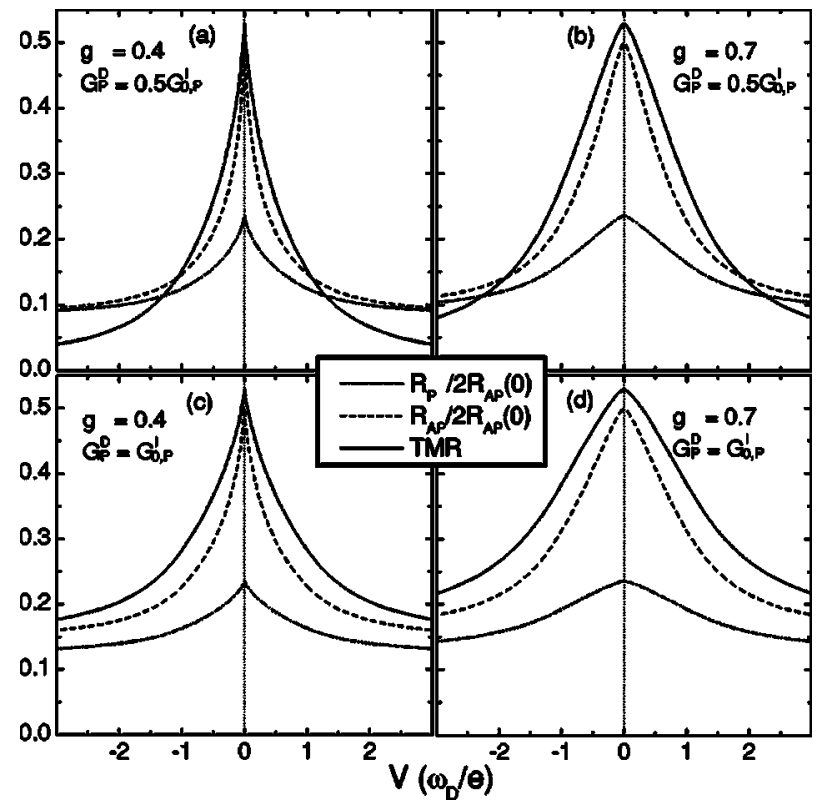

FIG. 2. Calculated results for $R_{\mathrm{P}}, R_{\mathrm{AP}}$, and TMR as functions of bias voltage for different $g$. The resistances are normalized by $2 R_{\mathrm{AP}}(V=0)$. Here, $E_{0}=E_{\mathrm{F}}$, and spin polarization $\left(\rho_{\uparrow}-\rho_{\downarrow}\right) /\left(\rho_{\uparrow}+\rho_{\downarrow}\right)$ $=0.6$.

$$
i B_{>}(\omega)=\int d t \exp \left\{i \omega t-g\left[\operatorname{Cin}\left(\omega_{\mathrm{D}} t\right)+i \operatorname{Si}\left(\omega_{\mathrm{D}} t\right)\right]\right\},
$$

where $\operatorname{Cin}(x)=\int_{0}^{x} d t[1-\cos (t)] / t$ and $\operatorname{Si}(x)=\int_{0}^{x} d t \sin (t) / t$ are the cosine and sine integral functions. ${ }^{31} G^{\mathrm{I}}(V)$ via impurities calculated by use of Eqs. (4) and (5) is plotted in Fig. 1 for nonmagnetic tunnel junctions. Since $\lim _{g \rightarrow 0}\left[i B_{>}(\omega)\right]$ $=2 \pi \delta(\omega), \lim _{g \rightarrow 0} G^{\mathrm{I}}(V)=G_{0}^{\mathrm{I}}$ is a constant independent of $V$, which is actually the maximum of $G^{\mathrm{I}}(V)$. From Fig. 1, it is clear that $G^{\mathrm{I}}(V)$ vanishes at $V=0$ for any nonzero $g$, indicating that tunneling via impurities is totally suppressed, and $G^{\mathrm{I}}(V)$ increases as the voltage $|V|$ is elevated. The stronger the electron-phonon coupling $g$ is, the slower the conductance $G^{\mathrm{I}}(V)$ increases with $|V|$.

To get some insight into low bias behavior of the tunneling conductance, we derive the low- $\omega$ approximation of Eq. (5),

$$
i \omega_{\mathrm{D}} B_{>}(\omega) \simeq \alpha \theta(\omega)\left(\omega / \omega_{\mathrm{D}}\right)^{-(1-g)},
$$

where $\alpha=2 \Gamma(1-g) \sin (g \pi) e^{-g \gamma}$, with $\gamma=0.5772$ the Euler constant and $\Gamma(z)=\int_{0}^{\infty} d t t^{z-1} e^{-t}$ is the ordinary gamma function. ${ }^{31}$ The unit step function $\theta(\omega)$ indicates that at zero temperature only emission of phonons is possible. Substituting Eq. (6) into Eq. (4), we obtain an interesting result $G^{\mathrm{I}}(V) \sim|V|^{2 g}$. For $g \leqslant 0.5(g>0.5)$, its slope $d G^{\mathrm{I}}(V) / d V$ $\sim|V|^{2 g} / V$ is discontinuous (continuous) at $V=0$.

In Fig. 2, the resistances for parallel alignment $\left(R_{\mathrm{P}}\right)$ and antiparallel alignment $\left(R_{\mathrm{AP}}\right)$ as well as TMR $=\left(R_{\mathrm{AP}}\right.$ $\left.-R_{\mathrm{P}}\right) / R_{\mathrm{AP}}$ of magnetic tunnel junctions are shown as functions of the bias voltage. It is found that $R_{\mathrm{AP}}$ always decreases faster than $R_{\mathrm{P}}$, resulting in a decline of TMR. This behavior can be understood from the following argument. At 
$V \simeq 0$, the direct tunneling dominates. For antiparallel alignment, the majority-spin (minority-spin) band in the left electrode is only connected to the minority-spin (majority-spin) band in the right electrode, leading to a relatively high resistance. With increasing $|V|$, the impurity tunneling increases. Electron hopping via the magnetic impurities causes a mixing of the two spin channels, which effectively connects up the majority-spin bands in the two electrodes. As a consequence, the resistance drops rapidly. For $g=0.4$, the TMR is discontinuous in slope at $V=0$. According to Fig. 2, either increasing the electron-phonon coupling $g$ or decreasing relatively the tunneling via impurities weakens the ZBA. In this theory, the energy scale of the ZBA is from zero to some $\omega_{\mathrm{D}}$. In view of the fact that $\omega_{\mathrm{D}}$ in most materials ranges from $10 \mathrm{meV}$ to $100 \mathrm{meV}$, the characteristic energies of the ZBA observed in tunnel junctions with $\mathrm{Ge},{ }^{1} \mathrm{NiO},{ }^{11}$ and AlAs (Ref. 12) as insulating barriers are consistent with our theory. For $\mathrm{FM} / \mathrm{Al}_{2} \mathrm{O}_{3} / \mathrm{FM}, V_{1 / 2}$ is greater than $100 \mathrm{meV} .{ }^{5-9}$ The impurity density might be low in these later junctions so that the ZBA is not prominent. According to a simplified model for impurity tunneling, ${ }^{32}$ we estimate that the impurity effect and the ZBA will become observable as the impurity number per unit cross section reaches $1 / \mu \mathrm{m}^{2}$ or higher.

The present theory as well as the earlier theories ${ }^{6,16-18,24}$ all predict that $G(V)$ for the tunnel junctions has a power-law dependence on small bias voltage, i.e., $G(V)-G(0) \sim|V|^{p}$. The low-temperature curves of the conductance (resistance) and TMR exhibit clear cusplike features at zero bias in most experiments, ${ }^{5-7,10,12}$ and noncusplike features in a few experiments. ${ }^{18}$ Mathematically, the cusplike (noncusplike) features require that the slope of the conductance $d G(V) / d V \sim|V|^{p} / V$ be discontinuous (continuous) at $V=0$, and so $p \leqslant 1 \quad(p>1)$. As mentioned in the Introduction, all the earlier theories ${ }^{16-18,24}$ predicted constant $p>1$, except for the theory of Zhang et al. ${ }^{6}$ where $p \equiv 1$. In the present theory, $p=2 g$ may be either smaller or greater than unity, depending on the strength of the impurity-mediated electron-phonon interaction, which is more flexible for understanding both the cusplike and noncusplike features within a unified theoretical framework. Our theory might be further verified directly by measurements of the isotope effect. Let us assume that the insulating barrier is made of a certain oxide. With a gradual replacement of the normal $\mathrm{O}^{16}$ atoms with isotope $\mathrm{O}^{18}$ atoms, the mass $M$ in a lattice cell increases, and the phonon frequency and Debye frequency change as $\omega_{q \lambda} \sim M^{-1 / 2}$ and $\omega_{\mathrm{D}} \sim M^{-1 / 2}$. We notice $\left|M_{q \lambda}\right|^{2} \sim\left(M_{\mathrm{I}} \omega_{q \lambda}\right)^{-1 \lambda} \sim M^{1 / 2}$, where $M_{\mathrm{I}}$ is the impurity mass rather than the cell mass. ${ }^{25,26}$ Consequently, the conductance exponent $2 g$ in the present theory will increase continuously as $2 g \sim M^{3 / 2}$ with the oxygen isotope enrichment. In contrast, the constant conductance exponents predicted by the earlier theories are expected not to change in this case.

D.Y.X. acknowledges the support of the National Natural Science Foundation of China under Grant No. 10374046. D.N.S was supported by ACS-PRF No. 36965-AC5, Research Corporation Grant No. CC5643, and the NSF Grants No. DMR-00116566 and No. DMR-0307170.
${ }^{1}$ M. Julliere, Phys. Lett. 54A, 225 (1975).

${ }^{2}$ S. Maekawa and U. Gäfvert, IEEE Trans. Magn. 18, 707 (1982).

${ }^{3}$ J. C. Slonczewski, Phys. Rev. B 39, 6995 (1989).

${ }^{4}$ T. Miyazaki and N. Tezuka, J. Magn. Magn. Mater. 139, 231 (1995).

${ }^{5}$ J. S. Moodera, L. R. Kinder, T. M. Wong, and R. Meservey, Phys. Rev. Lett. 74, 3273 (1995).

${ }^{6}$ S. Zhang, P. M. Levy, A. C. Marley, and S. S. P. Parkin, Phys. Rev. Lett. 79, 3744 (1997).

${ }^{7}$ J. S. Moodera, J. Nowak, and R. J. M. van de Veerdonk, Phys. Rev. Lett. 80, 2941 (1998).

${ }^{8}$ J. Zhang and R. M. White, J. Appl. Phys. 83, 6512 (1998).

${ }^{9}$ H. Boeve, E. Girgis, J. Schelten, J. De Boeck, and G. Borghs, Appl. Phys. Lett. 76, 1048 (2000).

${ }^{10}$ R. Jansen and J. S. Moodera, Phys. Rev. B 61, 9047 (2000).

${ }^{11}$ E. Y. Tsymbal, A. Sokolov, I. F. Sabirianov, and B. Doudin, Phys. Rev. Lett. 90, 186602 (2003).

${ }^{12}$ R. Mattana, J.-M. George, H. Jaffrès, F. Nguyen Van Dau, A. Fert, B. Lépine, A. Guivarc'h, and G. Jézéquel, Phys. Rev. Lett. 90, 166601 (2003).

${ }^{13}$ H. F. Ding, W. Wulfhekel, J. Henk, P. Bruno, and J. Kirschner, Phys. Rev. Lett. 90, 116603 (2003).

${ }^{14}$ A. M. Bratkovsky, Phys. Rev. B 56, 2344 (1997).

${ }^{15}$ L. Sheng, Y. Chen, H. Y. Teng, and C. S. Ting, Phys. Rev. B 59, 480 (1999).
${ }^{16}$ F. Guinea, Phys. Rev. B 58, 9212 (1998).

${ }^{17}$ E. Y. Tsymbal and D. G. Pettifor, J. Appl. Phys. 85, 5801 (1999).

${ }^{18}$ A. M. Bratkovsky, Appl. Phys. Lett. 72, 2334 (1998).

${ }^{19}$ E. Y. Tsymbal and D. G. Pettifor, Phys. Rev. B 58, 432 (1998); 64, 212401 (2001); E. Y. Tsymbal, V. M. Burlakov, and I. I. Oleinik, ibid. 66, 073201 (2002).

${ }^{20}$ R. Jansen and J. C. Lodder, Phys. Rev. B 61, 5860 (2000).

${ }^{21}$ A. Vedyayev, D. Bagrets, A. Bagrets, and B. Dieny, Phys. Rev. B 63, 064429 (2001).

${ }^{22}$ J. Inoue, N. Nishimura, and H. Itoh, Phys. Rev. B 65, 104433 (2002).

${ }^{23}$ H. Sun, K. W. Yu, and Z. Y. Li, Phys. Rev. B 68, 054413 (2003).

${ }^{24}$ L. I. Glazman and K. A. Matveev, Zh. Eksp. Teor. Fiz. 94, 232 (1988) [Sov. Phys. JETP 67, 1276 (1988)].

${ }^{25}$ S. Koshino, Prog. Theor. Phys. 24, 484 (1960); 24, 1049 (1960); P. L. Taylor, Proc. Phys. Soc. London 80, 755 (1962); Phys. Rev. 135, A1333 (1964).

${ }^{26}$ G. D. Mahan, Many-Particle Physics (Plenum Press, New York, 1990).

${ }^{27}$ C. Caroli, R. Combescot, P. Nozieres, and D. Saint-James, J. Phys. C 5, 21 (1972); C. B. Duke, Tunneling in Solids (Academic Press, New York, 1969).

${ }^{28}$ I. G. Lang and Y. A. Firsov, Zh. Eksp. Teor. Fiz. 43, 1843 (1962) [Sov. Phys. JETP 16, 1301 (1962)]. 
${ }^{29}$ A. R. Hibbs and R. P. Feynman, Quantum Methanics and Path Integrals (McGraw-Hill, New York, 1965); K. C. Chou, Z. B. Su, B. L. Hao, and L. Yu, Phys. Rep. 118, 1 (1985).

${ }^{30}$ For the path-integral approach to double-barrier tunneling, see L. Y. Chen and C. S. Ting, Phys. Rev. B 41, 8533 (1990); Phys.
Rev. Lett. 64, 3159 (1990).

${ }^{31}$ I. S. Gradshteyn and I. M. Ryzhik, Tables of Integrals, Series, and Products (Academic Press, San Diego, 2000).

${ }^{32}$ L. Sheng, D. Y. Xing, and D. N. Sheng, Phys. Rev. B 69, 132414 (2004). 